\title{
Prevalência de lesões orofaciais e o nível de conhecimento sobre proteção bucal em uma equipe brasileira de judô
}

\author{
Prevalence of orofacial injuries and the level of knowledge about oral protection in a brazilian judo
}

team

Prevalencia de lesiones orofaciales y nivel de conocimiento sobre protección oral en un equipo judo
brasileño

Recebido: 15/05/2021 | Revisado: 23/05/2021 | Aceito: 27/05/2021 | Publicado: 10/06/2021

\author{
Felipe Melo de Araújo \\ ORCID: https://orcid.org/0000-0001-6237-3548 \\ Universidade Federal do Rio de Janeiro, Brasil \\ E-mail: felipe_meloaraujo@hotmail.com \\ Tiago Braga Rabello \\ ORCID: https://orcid.org/0000-0003-2502-9611 \\ Universidade Federal do Rio de Janeiro, Brasil \\ E-mail: tiagobragarabello@gmail.com \\ Lucas Thomazotti Berard \\ ORCID: https://orcid.org/0000-0003-1250-4970 \\ Universidade de São Paulo, Brasil \\ E-mail: lucas.berard@usp.br \\ Neide Pena Coto \\ ORCID: https://orcid.org/0000-0002-3235-0684 \\ Universidade de São Paulo, Brasil \\ E-mail: npcoto@usp.br \\ Kátia Regina Hostilio Cervantes Dias \\ ORCID: https://orcid.org/0000-0002-8133-2394 \\ Universidade Federal do Rio de Janeiro, Brasil \\ E-mail: cervantes.dias@gmail.com
}

\begin{abstract}
Resumo
Os atletas das modalidades que envolvem contato estão frequentemente sujeitos a traumas orofaciais, principalmente em esportes de combate como o judô. Porém, a utilização dos protetores bucais pode minimizar a ocorrência dos traumatismos e evitar lesões orofaciais decorrentes da prática esportiva. O objetivo desse trabalho foi determinar a prevalência de lesões orofaciais nos atletas das equipes de base da seleção brasileira de judô, bem como, avaliar o nível de conhecimento dos mesmos sobre o uso de protetores bucais. Foi aplicado um questionário estruturado, com 10 perguntas específicas para 46 atletas das seleções de base de judô, masculina e feminina do Brasil, referentes ao tema da pesquisa. Os resultados sugerem que a maioria dos atletas já sofreu algum tipo de traumatismo orofacial durante a prática do judô e a maior parte dos atletas não utiliza o protetor bucal, por diversos fatores. Conclui-se que os traumas orofaciais mostraram alta prevalência sendo que lacerações de lábio, língua e mucosa jugal foram os mais relatados pelos atletas de judô da seleção brasileira e o nível de conhecimento dos mesmos sobre o uso de protetores bucais se mostrou baixo, apontando a necessidade de ações informativas sobre esse assunto entre os atletas da seleção brasileira de judô.
\end{abstract}

Palavras-chave: Atletas; Protetores bucais; Traumatismos.

\begin{abstract}
Contact athletes are often subject to orofacial trauma, especially in combat sports such as judo. However, the use of mouthguards can minimize the occurrence of trauma and prevent orofacial injuries resulting from sports. The objective of this work was to determine the prevalence of orofacial injuries in the athletes of the basic teams of the Brazilian judo team, as well as to evaluate their level of knowledge about the use of mouth protectors. A structured questionnaire was applied, with 10 specific questions for 46 athletes from the basic judo teams, male and female in Brazil, referring to the research theme. The results suggest that most athletes have already suffered some type of orofacial trauma during the practice of judo and most athletes do not use mouthguards, due to several factors. It is concluded that orofacial traumas showed a high prevalence, with lacerations of the lip, tongue and cheek mucosa being the most reported by judo athletes of the Brazilian team and their level of knowledge about the use of mouthguards was low, pointing to the need for informational actions on this subject among athletes of the Brazilian judo team.
\end{abstract}


Keywords: Athletes; Injuries; Mouth protectors.

\section{Resumen}

Los atletas de contacto a menudo están sujetos a traumatismos orofaciales, especialmente en deportes de combate como el judo. Sin embargo, el uso de protectores bucales puede minimizar la aparición de traumatismos y prevenir lesiones orofaciales resultantes de los deportes. El objetivo de este trabajo fue determinar la prevalencia de lesiones orofaciales en los deportistas de los equipos básicos de la selección brasileña de judo, así como evaluar su nivel de conocimiento sobre el uso de protectores bucales. Se aplicó un cuestionario estructurado, con 10 preguntas específicas para 46 deportistas de los equipos básicos de judo, masculinos y femeninos de Brasil, sobre el tema de investigación. Los resultados sugieren que la mayoría de los deportistas ya han sufrido algún tipo de trauma orofacial durante la práctica del judo y la mayoría de los deportistas no utilizan protectores bucales, debido a varios factores. Se concluye que los traumatismos orofaciales mostraron una alta prevalencia, siendo las laceraciones de labio, lengua y mucosa yugal las más reportadas por los judoistas de la selección brasileña y su nivel de conocimiento sobre el uso de protectores bucales fue bajo, señalando la necesidad de información acciones sobre este tema entre los deportistas de la selección brasileña de judo.

Palabras clave: Atletas; Protectores bucales; Traumatismos.

\section{Introdução}

Lesões orofaciais são representadas por traumatismo dentário (fratura, luxação e avulsão), laceração de tecidos moles (Galic et al., 2018; Tewari et al., 2021), fraturas dos ossos da face e lesões da articulação temporomandibular (Emerich, Kaczmarek, 2010; Oliveira et al., 2020). Atletas que praticam esportes de combate como o judô apresentam maior risco de sofrerem lesões orofaciais do que atletas de esportes de categorias sem contato (Tulunoglu \& Ozbek, 2006; Glendor et al., 2009; McBain et al., 2012; Schildknecht et al., 2012). Sabe-se que 31,0\% das lesões orofaciais são decorrentes de traumas durante a prática de esportes, representando quase um terço de todas as lesões faciais ocorridas, sendo metade intraorais (Keçeci et al., 2005).

No entanto, é possível evitar lesões orofaciais na prática esportiva reduzindo consideravelmente a sua ocorrência, por meio do uso de protetores bucais (Coto et al., 2012; Vucic et al., 2016).

Os protetores bucais são dispositivos intraorais que atuam na absorção e dissipação da energia transmitida durante choques entre os atletas e/ou acessórios da prática do esporte (Gialain et al., 2016) além de impedir o contato traumático entre os arcos dentários antagonistas (Inouye \& McGrew, 2015). Os protetores bucais podem ser encontrados no mercado recebendo o nome de pré-fabricados, porém, são contra-indicados; e protetores individualizados, confeccionados pelo cirurgião dentista sobre modelo em gesso do arco superior do atleta, permitem ingestão de líquidos e comunicação durante a prática do esporte, são bem ajustados dando conforto e segurança ao atleta (Gialain,2016).

Visto que o judô é um dos esportes mais praticados no mundo, cerca de 20 milhões de praticantes, segundo a Confederação Brasileira de Judô (CBJ) (Nunes \& Rubio, 2012). Que nem sempre o atleta dessa modalidade utiliza protetores bucais para esporte, havendo maior exposição a ocorrência de lesões orofaciais durante treinos e competições, é necessário entender como esse atleta vê a proteção bucal e conhecer a prevalência das lesões orofaciais ocorridas durante a prática de esporte. Essa ação trará orientações capazes de minimizar os riscos de qualquer lesão orofacial que pode impactar em seu desempenho (Ribeiro et al., 2021).

Nesse contexto, o objetivo deste estudo foi determinar a prevalência de lesões orofaciais nos atletas das equipes de base da seleção brasileira de judô, bem como, avaliar o nível de conhecimento dos mesmos sobre o uso de protetores bucais.

\section{Metodologia}

Foi aplicado um questionário estruturado (Tabela 1), com 10 perguntas específicas para 46 atletas das seleções de base de judô, masculina e feminina do Brasil, referentes ao tema da pesquisa. A aplicação do questionário foi individual, durante os treinos dos atletas. Após leitura do TCLE, a concordância dos termos e as devidas assinaturas, uma cópia do termo 
de consentimento livre e esclarecido foi entregue ao atleta juntamente com o questionário. A pesquisa possui inscrição no Comitê de Ética em Pesquisa do Hospital Universitário Clementino Fraga Filho/Universidade Federal do Rio de Janeiro (CAAE: 64088016.5.0000.5257), estando de acordo com todas as normas vigentes em pesquisa com seres humanos.

Os dados obtidos foram analisados determinando uma análise descritiva.

Tabela 1. Questionário.

\begin{tabular}{lll}
\hline 1- Nome: & 2- Data de Nascimento & 3- Idade: (anos) \\
\hline $\begin{array}{l}\text { 4- Há quantos anos pratica o } \\
\text { judôo? }\end{array}$ & 5 - Categoria: $(\mathrm{kg})$ & \\
\hline 6- Grau de escolaridade & $(\quad)$ ensino fundamental (até o atual $9^{\circ}$. & $\left(\begin{array}{l}\text { ) ensino médio incompleto } \\
\text { Ano) }\end{array}\right.$ \\
\hline & $(\quad)$ ensino médio completo & $\left(\begin{array}{l}\text { superior incompleto } \\
\text { pós graduação latu sensu ou }\end{array}\right.$ \\
\hline & $(\quad)$ superior completo & $\begin{array}{l}\text { strictu sensu (mestrado e/ou } \\
\text { doutorado) }\end{array}$
\end{tabular}

7- Já sofreu algum trauma
na face praticando o judô?
$\begin{array}{ll}\text { ( ) sim } & (\quad \text { ) não }\end{array}$

Caso SIM, qual ou quais?

( ) laceração de lábio ( ) laceração de bochecha

( ) fratura dentária ( ) laceração de língua

\begin{tabular}{|c|c|c|}
\hline $\begin{array}{l}\text { 8- Já usou algum tipo de } \\
\text { protetor bucal durante o } \\
\text { treino, mesmo não utilizando } \\
\text { durante a competição? } \\
\left(\begin{array}{l}) \text { sim } \\
(\quad) \text { não }\end{array}\right.\end{array}$ & Se SIM, qual o tipo de protetor bucal? & $\begin{array}{l}\text { ( ) "ferve e morde" } \\
\text { ( ) feito por um dentista }\end{array}$ \\
\hline $\begin{array}{l}\text { 9- Já recebeu alguma } \\
\text { informação sobre protetor } \\
\text { bucal? } \\
\begin{array}{lll}(\quad) \operatorname{sim} & (\quad) \text { não }\end{array}\end{array}$ & $\begin{array}{l}\text { Caso SIM, por quem? } \\
(\quad) \text { dentista }\end{array}$ & ( ) preparador físico \\
\hline
\end{tabular}

\section{0- Caso a Federação}

Internacional

incentivasse a utilização, Caso NÃO, qual seria o motivo?

tentaria utilizar visando a

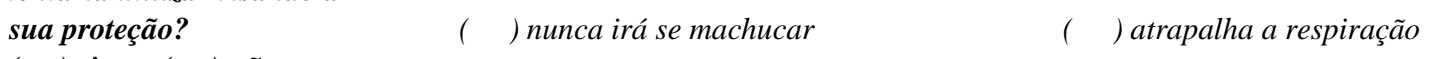

( ) $\operatorname{sim}(\quad)$ não

\begin{tabular}{|c|c|c|}
\hline( & ( ) falta informação & ( ) acha que não adianta nada \\
\hline & ( ) acha incômodo & $\begin{array}{l}\text { ( ) atrapalha no desempenho do } \\
\text { atleta }\end{array}$ \\
\hline & ( ) preço elevado & \\
\hline
\end{tabular}

Fonte: Autores. 


\section{Resultados}

Dos entrevistados, $43,0 \%$ eram do sexo masculino e $57,0 \%$ do sexo feminino. Os atletas apresentaram idade variando de 18 a 30 anos, com predominância de 18 a 20 anos, correspondendo a $91 \%$ da amostra. A média de idade dos atletas foi de 19,1 anos.

Em relação ao tempo de prática no esporte, a variação foi de 6 a 22 anos, com maior concentração entre 7 e 13 anos de prática, cerca de 79,0\%. O Gráfico 1 mostra a resposta do questionamento se já sofreu trauma orofacial na prática do judô. As respostas foram dicotômicas.

Gráfico 1. Prevalência de trauma na região orofacial durante a prática de judô em atletas da equipe brasileira.

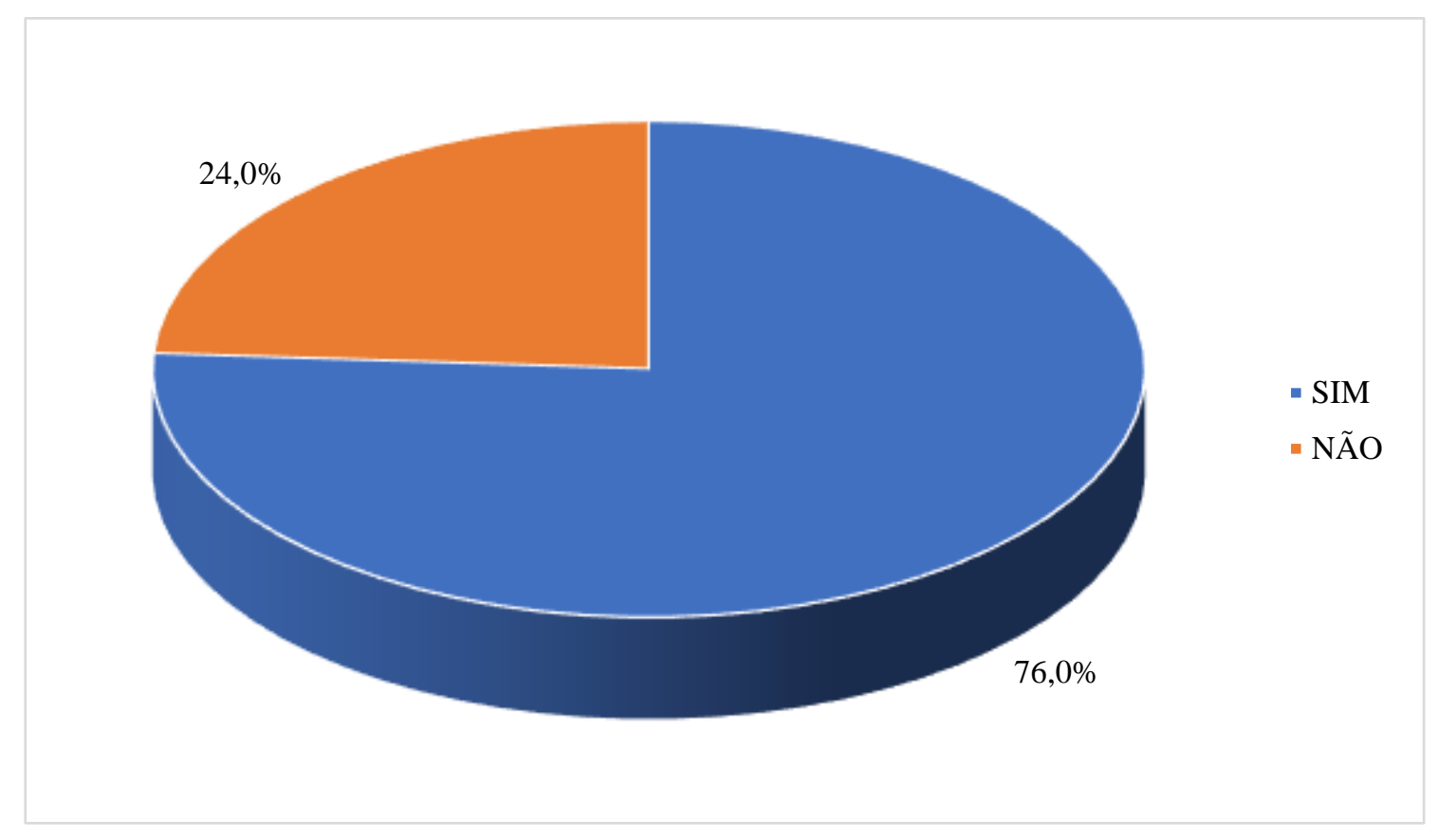

Fonte: Autores.

O Gráfico 2 mostra que tipo de lesão orofacial ocorreu nos atletas que responderam "sim" para a pergunta anterior. 
Research, Society and Development, v. 10, n. 6, e57810616133, 2021

(CC BY 4.0) | ISSN 2525-3409 | DOI: http://dx.doi.org/10.33448/rsd-v10i6.16133

Gráfico 2. Tipos de traumas orofaciais relatados pelos atletas durante a prática de judô.

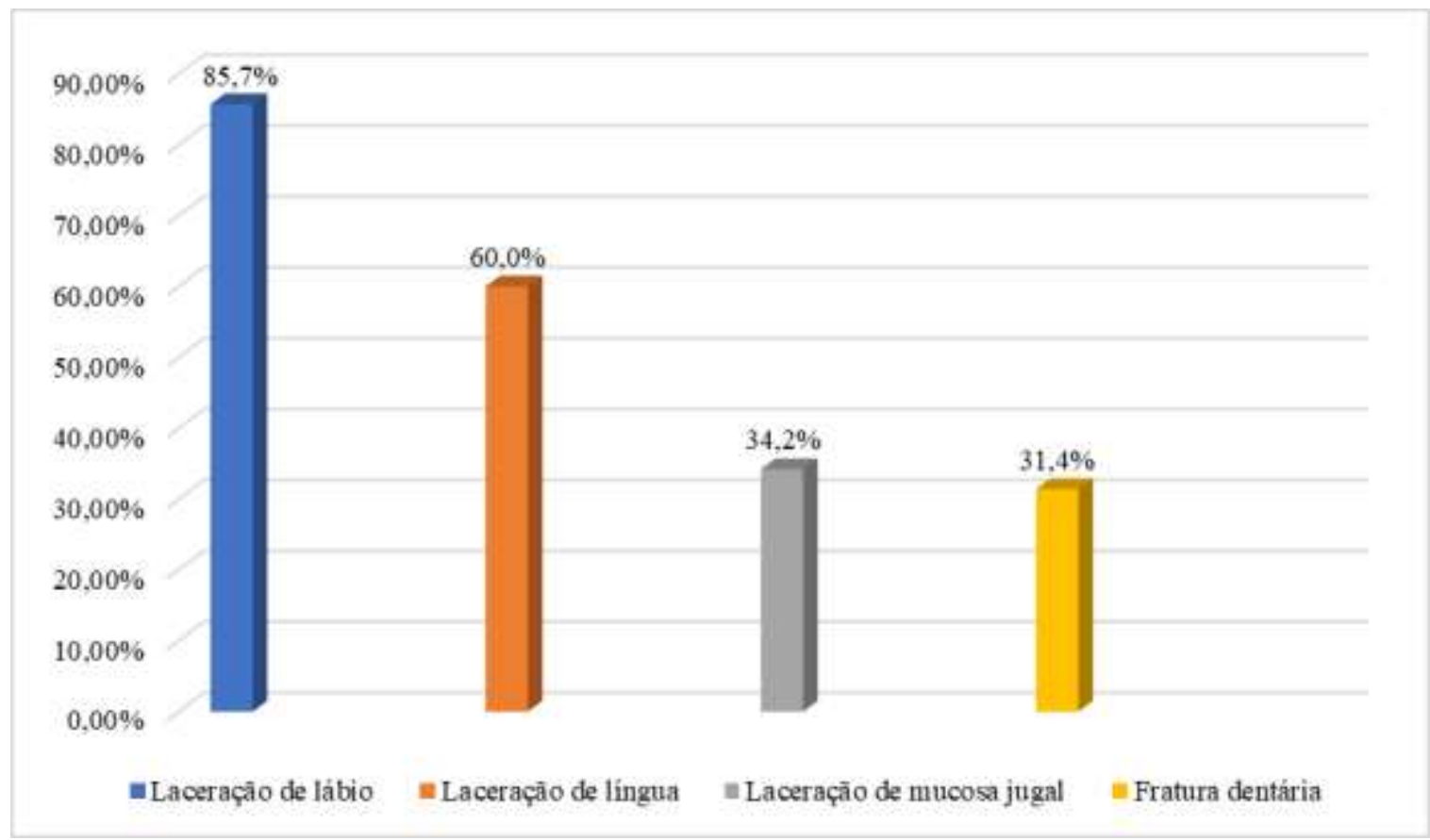

Fonte: Autores.

O Gráfico 3 traz o nível de informação e conhecimento sobre protetor bucal que o atleta apresentou, com resposta dicotômica ("sim/não"). Dos que responderam "sim", foi solicitado que relatassem a fonte da informação, mostrado no Gráfico 4.

Gráfico 3. Nível de conhecimento dos praticantes de judô da seleção brasileira sobre o uso de protetores bucais para a prática do esporte.

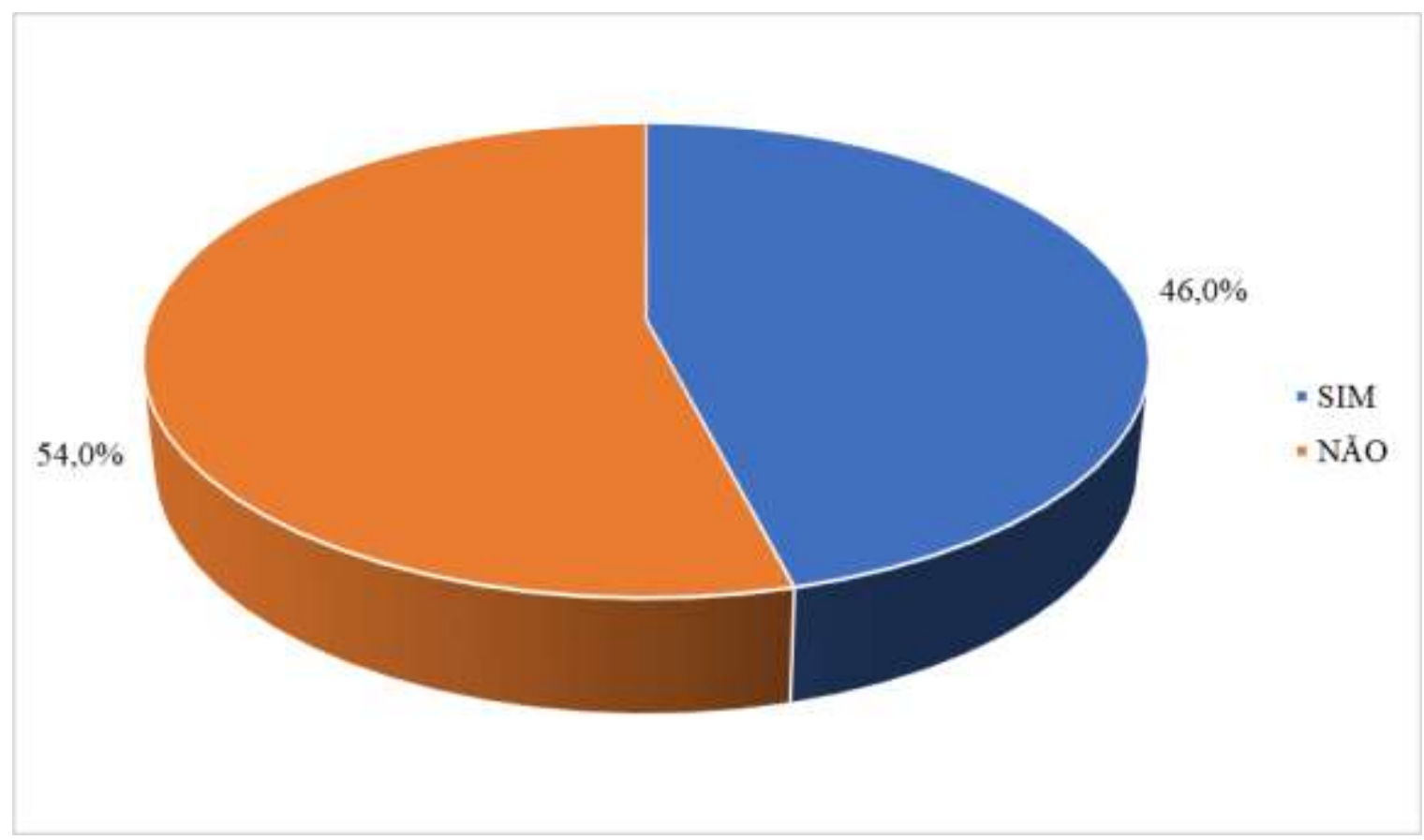

Fonte: Autores. 
Gráfico 4. Principais veículos informativos acerca da necessidade do uso de protetores bucais na prática do esporte.

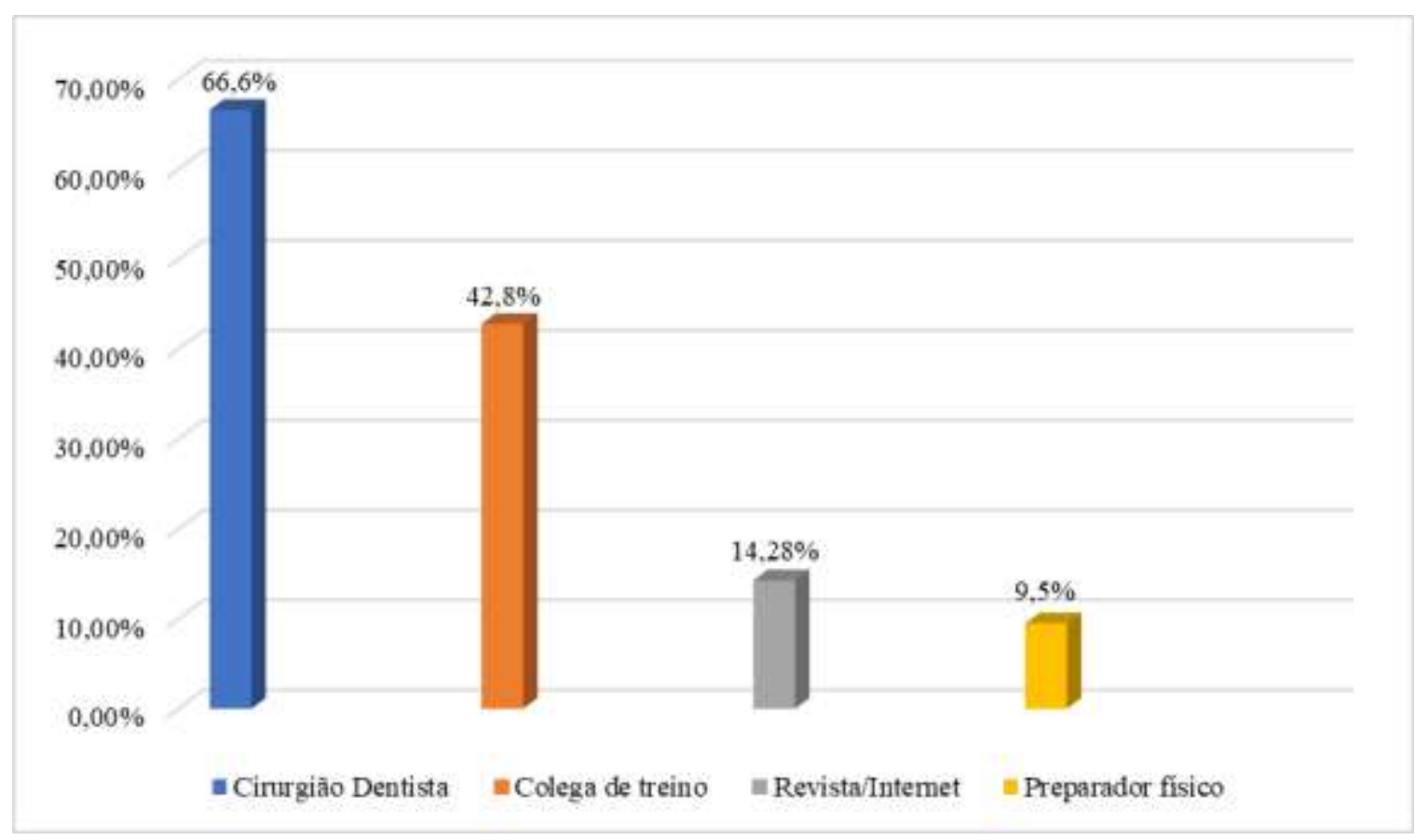

Fonte: Autores.

Em outra pergunta foi questionado se estes atletas utilizam o protetor bucal para esporte, sendo que, $85,0 \%$ relataram que não utilizam.

De toda amostra, apenas 3 atletas $(6,5 \%)$ utilizaram um protetor bucal confeccionado por um cirurgião-dentista. 93,5\% de todos os entrevistados nunca utilizaram protetor individualizado confeccionado pelo cirurgião dentista.

O Gráfico 5 mostra o quanto o atleta estaria disposto a utilizar o protetor bucal, caso sua Federação incentivasse.

Gráfico 5. Prevalência do uso do protetor bucal quando há incentivo por parte dos gestores e dirigentes esportivos.

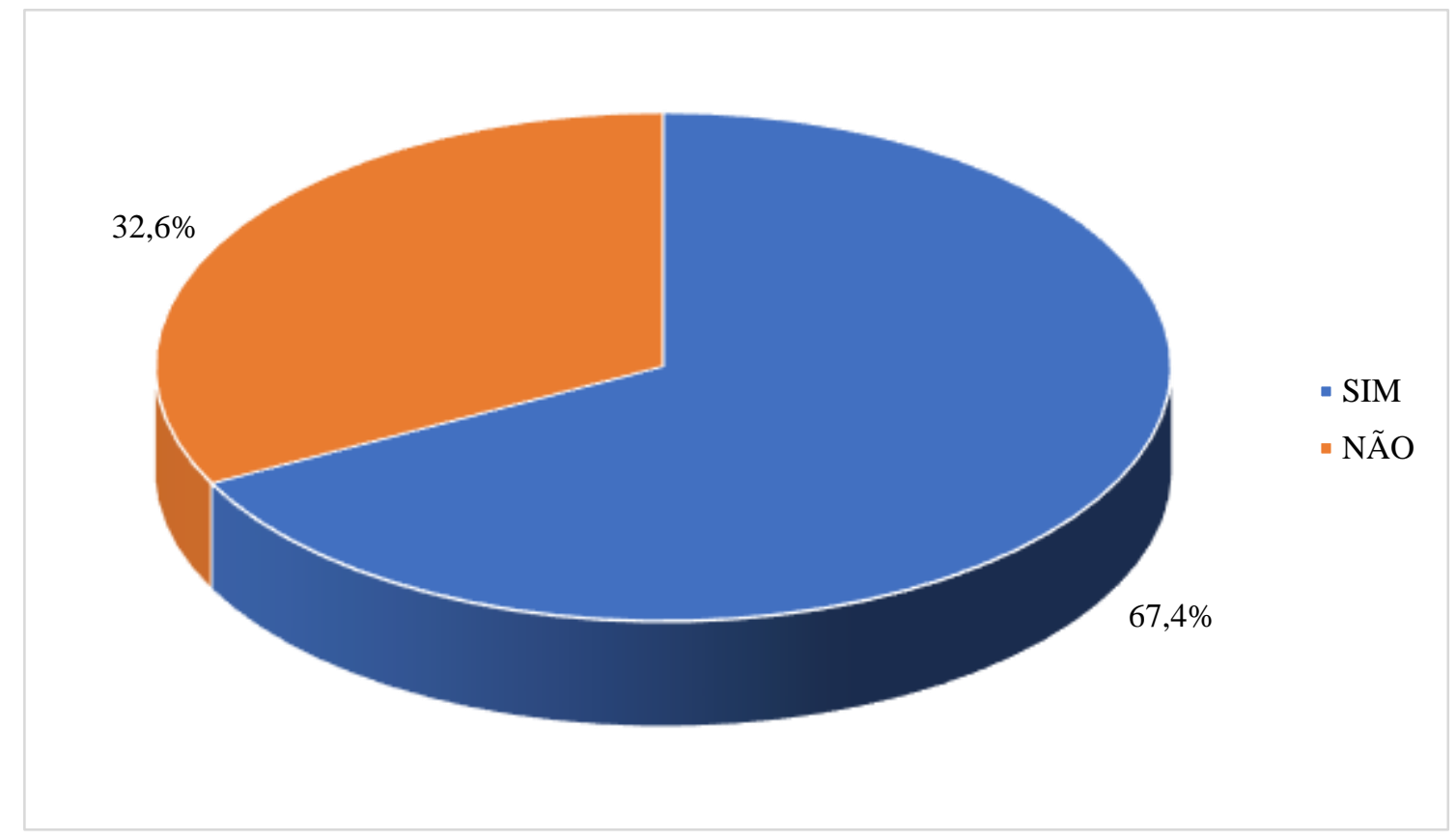

Fonte: Autores. 
Para os 15 atletas que relataram a não utilização em caso de incentivo por parte dos dirigentes e gestores esportivos foi questionado o motivo, que pode ser observado no Gráfico 6.

Gráfico 6. Motivos apontados pelos atletas para a não utilização do protetor bucal na prática do esporte.

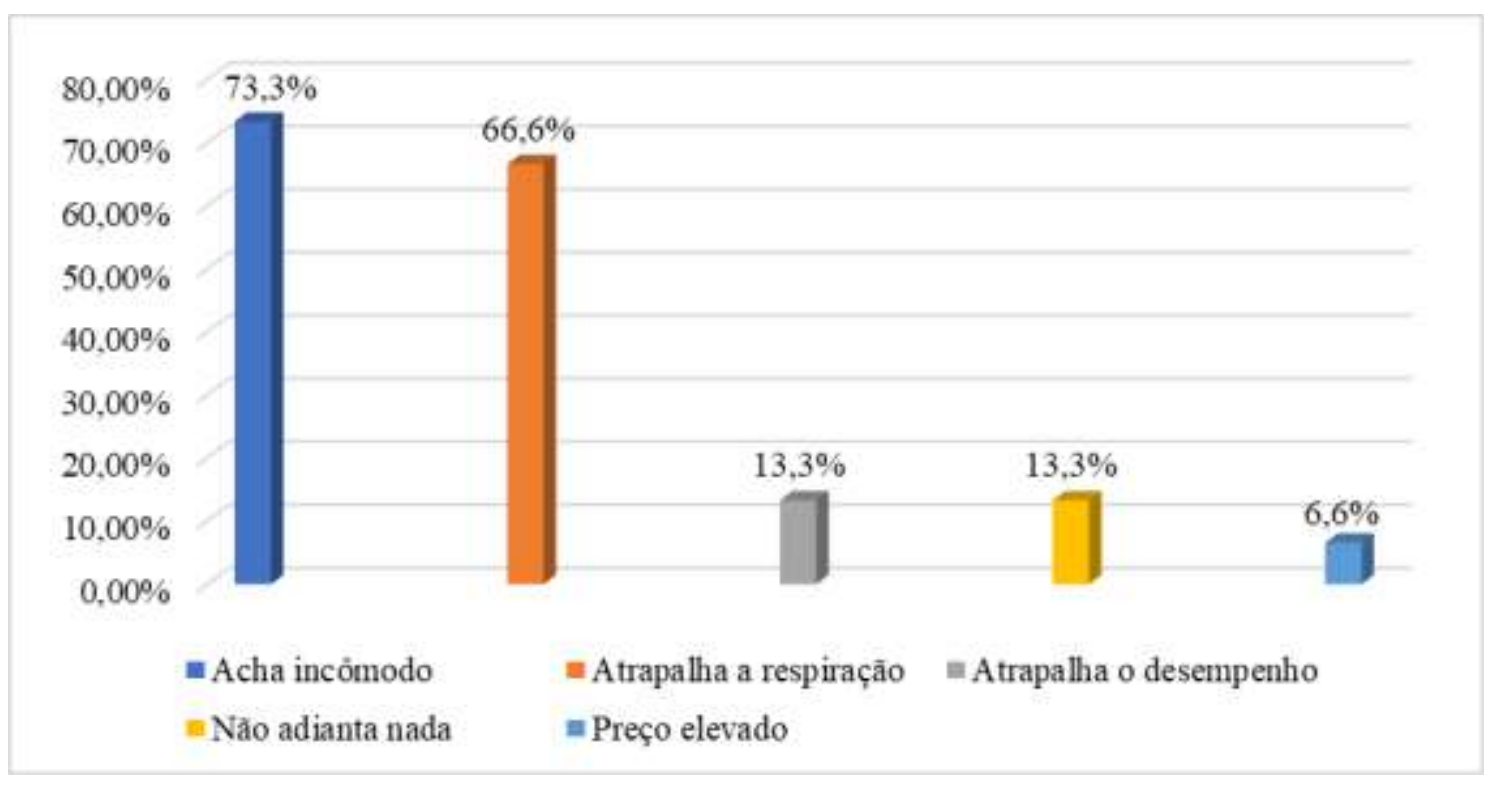

Fonte: Autores.

\section{Discussão}

As Lesões orofaciais decorrentes da prática esportiva vêm aumentando substancialmente (Fronza et al., 2020). A prevalência de lesões orofaciais em esportes de combate é de aproximadamente 30,0\% (Polmann et al., 2019). No presente estudo, foi encontrado um percentual duas vezes maior de trauma em atletas de judô como também superior ao encontrado no levantamento realizado por Caciones et al., 2018, com atletas profissionais e amadores de Boxe, também elencado como esporte de combate, onde houve uma prevalência de $44,4 \%$ de lesões orofaciais. Quanto à utilização de protetores bucais, no presente estudo, poucos atletas utilizam o dispositivo de proteção, ao contrário de Cavalcanti et al., 2012, relatou que 50,0\% dos atletas de judô utilizam protetor bucal. Porém, quando uma modalidade impõe, seu uso é obrigatório e esses números chegam a próximo de 90,0\%, como relatou Caciones et al. em 2018.

Dentre os atletas desta pesquisa que já sofreram trauma orofacial praticando judô, a prevalência foi maior em laceração de lábio, língua, mucosa jugal, seguida por fraturas dentárias. No estudo de Siewe et al., 2015, a prevalência de laceração labial em praticantes do judô foi de 11,4\%, diferença essa explicada pelo fato de que neste último estudo os sujeitos da pesquisa não eram atletas de seleção, portanto não praticavam o judô com mesma frequência que os indivíduos do presente estudo.

Quando o questionamento foi sobre protetor bucal, no presente estudo foi encontrado que menos da metade dos atletas relataram ter conhecimento sobre a necessidade do uso desse dispositivo de proteção. Este conhecimento foi inferior ao encontrado no trabalho de Ferrari \& Medeiros, 2002, onde o grau de conhecimento desse quesito entre os atletas de judô foi de $61,7 \%$.

Dos atletas do presente estudo que referiram conhecer sobre o protetor bucal, sendo que a informação foi adquirida por meio do Cirurgião Dentista, por colega de treino, por revista/internet e pelo preparador físico, em ordem decrescente da fonte. No estudo de Padilha et al., 2021, mais de 25,0\% dos atletas de Rugby relataram que foram orientados a utilizar o 
protetor bucal por diversas fontes, como internet, parentes e outros profissionais da saúde e mais de $74 \%$ foram instruídos pelo preparador físico, educador físico ou treinador.

Uma das perguntas realizadas aos atletas era que, caso a Federação Internacional de judô incentivasse o uso do protetor bucal, se eles o utilizariam. Porém, o que mais chama a atenção é que dos atletas que se posicionaram contrários ao uso, alguns nunca utilizaram qualquer protetor bucal e o restante utilizou um protetor tido como inadequado, "pré fabricado", refletindo o baixo grau de conhecimento e a necessidade de se investir na educação e conscientização dos atletas., De acordo com Parker et al., 2016, em 95,0\% dos casos em que os protetores bucais não foram utilizados nos esportes de contato, o principal motivo foi a falta de conhecimento, e por julgar desnecessário, 73,0\% dos atletas não utilizam o dispositivo de proteção. Dados do presente estudo foram semelhantes aos achados de Tiryaki et al., 2017, segundo o qual, 7,6\% dos atletas alegaram não utilizar o protetor bucal pelo alto custo, enquanto que, no estudo em questão esse fato foi reportado por $6,6 \%$ atletas.

É fundamental investir na prevenção e utilização de aparatos de proteção como o protetor bucal, na medida em que, inúmeros problemas oriundos do traumatismo poderiam ser evitados ou minimizados. Além de lesões físicas, fatores psicológicos e sociais são citados como consequências das lesões orofaciais (Tuna \& Ozel, 2014). O estudo de Tiwari et al., 2014, demonstrou que atletas que não usavam protetores bucais tiveram mais lesões orofaciais quando comparados aos atletas que utilizaram. A criação de um sistema de vigilância, buscando evitar a lesão do atleta e consequente afastamento dos treinos e competições é sugerida por Pocecco et al., 2013, corroborando com a crescente necessidade da prevenção.

Este estudo sugere uma alta prevalência de traumatismos orofaciais em atletas de judô e evidencia a necessidade da prevenção dessas lesões, a partir da utilização de protetores bucais. Esses achados encorajam novas pesquisas, relacionando o tipo de protetor bucal utilizado às diferentes lesões orofaciais sofridas por essa população, bem como, a atuação de outros profissionais de áreas integradas, a fim de difundir a importância da prevenção e promoção de saúde oral do paciente atleta, especialmente nos esportes de combate.

\section{Conclusão}

Os resultados deste estudo demonstram que:

Os traumas orofaciais mostraram alta prevalência sendo que lacerações de lábio, língua e mucosa jugal foram os mais relatados pelos atletas de judô da seleção brasileira.

O nível de conhecimento dos mesmos sobre o uso de protetores bucais se mostrou baixo, apontando a necessidade de ações informativas sobre esse assunto entre os atletas da seleção brasileira de judô.

\section{Referências}

Bergman, L., Milardović Ortolan, S., Žarković, D., Viskić, J., Jokić, D., \& Mehulić, K. (2017). Prevalence of dental trauma and use of mouthguards in professional handball players. Dental Traumatology, 33(3), 199-204.

Boffano, P., Boffano, M., Gallesio, C., Roccia, F., Cignetti, R., \& Piana, R. (2012). Rugby athletes' awareness and compliance in the use of mouthguards in the North West of Italy. Dental Traumatology, 28, 210-3.

Caciones, A., Antunes, M., Assunção, V., Albuquerque, T., \& Luís H. (2018). Estudo preliminar da saúde oral de desportistas praticantes de boxe. Rev Port Estomatol Cir Maxilofac, 59, 215-20.

Cavalcanti, A., Santos, F. G., Peixoto, L. R., Gonzaga, A., Dias, C., \& Xavier, A. (2012). Orofacial injuries in combat sports practitioners. Pesqui Bras Odontopediatria Clin Integr, 12, 223-8.

Coto, N. P., Brito e Dias, R., Costa, R. A., Antoniazzi, T. F., \& de Carvalho, E. P. (2007). Mechanical behavior of ethylene vinyl acetate copolymer (EVA) used for fabrication of mouthguards and interocclusal splints. Braz Dent J, 18(4), 324-8.

Coto, N. P., Meira, J. B. C., e Dias, R. B., Driemeier, L., de Oliveira Roveri, G., \& Noritomi, P. Y. (2012). Assessment of nose protector for sport activities: finite element analysis. Dental traumatology, 28(2), 108-113. 
Emerich, K., \& Kaczmarek, J. (2010). First aid for dental trauma caused by sports activities:state of knowledge, treatment and prevention. Sports Med, 40, 361-366.

Eroje, A. B., Tikare, S., Al Qahtani, N. A., Braimoh, O. B., Sundarraj, R. K., \& Muteq, M. A., et al. (2020). Orofacial trauma awareness among sports teachers in Southern Saudi Arabia. Niger J Clin Pract, 23, 343-8.

Ferrari, C. H., \& Medeiros, J. M. F. (2002). Dental trauma and level of information: mouthguard use in different contact sports. Dental Traumatology, 18(3), 144-147.

Fronza, H. P., Stolf, S. C., Taguchi, C. M. C., Coto, N. P., \& Padilha, A. C. L. (2020). Determinants for traumatic orofacial injuries in sport: Extrinsic factors in a scoping review. Dental Traumatology, 36(6), 598-606.

Galic, T., Kuncic, D., Poklepovic Pericic, T., Galic, I., Mihanovic, F., Bozic, J., \& Herceg, M. (2018). Knowledge and attitudes about sports-related dental injuries and mouthguard use in young athletes in four different contact sports-water polo, karate, taekwondo and handball. Dental Traumatology, 34(3), 175181.

Gialain, I. O., Coto, N. P., Driemeier, L., Noritomi, P. Y., \& Dias, R. B. (2016). A three-dimensional finite element analysis of the sports mouthguard. Dental Traumatology, 32(5), 409-15.

Glendor, U. (2009). A etiology and risk factors related to traumatic dental injuries-a review of the literature. Dental Traumatology, 25, 19-31.

Ilia, E., Metcalfe, K., \& Heffernan, M. (2014). Prevalence of dental trauma and use of mouthguards in rugby union players. Aust Dent J, 59, $473-81$. Inouye, J., \& McGrew, C. (2015). Dental Problems in Athletes. Curr Sports Med Rep, 14(1), 27-33.

Keçeci, A. D., Eroglu, E., \& Baydar, M. L. (2005). Dental trauma incidence and mouthguard use in elite athletes in Turkey. Dental Traumatology, 21, 76-9.

McBain, K., Shrier, I., Shultz, R., Meeuwisse, W. H., Klügl, M., Garza, D., \& Matheson, G.O. (2012). Prevention of sports injury I: a systematic review of applied biomechanics and physiology outcomes research. Br J Sports Med, 46, 169-73.

Nunes, A. V., \& Rubio, K. (2012). As origens do judô brasileiro: a árvore genealógica dos medalhistas olímpicos. Revista Brasileira de Educação Física e Esporte, 26(4), 667-678.

Oliveira Werlich, M., Honnef, L. R., Silva Bett, J. V., Domingos, F. L., Pauletto, P., Dulcineia Mendes de Souza, B., Mageste Duque, T., Curi Hallal, A.L., \& De Luca Canto, G. (2020). Prevalence of dentofacial injuries in contact sports players: A systematic review and meta-analysis. Dental Traumatology, 36(5), 477-488.

Padilha, A. C. L., Constante, H. M., Fronza, H. P., \& Coto, N. P. (2021). Orofacial trauma and mouthguard use in Brazilian rugby union players. Dental Traumatology, 37(1), 53-57.

Parker, K., Marlow, B., Patel, N., \& Gill, D. (2016). An audit on the use of mouthguards in orthodontic patients before treatment. BOS Clinical Effectiveness Bulletin, 36, 14-16.

Parker, K., Marlow, B., Patel, N., \& Gill, D. S. (2017). A review of mouthguards: effectiveness, types, characteristics and indications for use. Brit Dent J, 222, 629-633.

Pocecco, E., Ruedl, G., Stankovic, N., Sterkowicz, S., Del Vecchio, F.B., Gutiérrez-García, C., Rousseau, R., Wolf, M., Kopp, M., Miarka, B., Menz, V., Krusmann, P., Calmet, M., Malliaropoulos, N., \& Burtscher, M. (2013). Injuries in judo: a systematic literature review including suggestions for prevention. British journal of sports medicine, $47(18), 1139-1143$.

Polmann, H., Melo, G., Conti Réus, J., Domingos, F. L., de Souza, B. D. M., Padilha, A. C., Duque, T. M., Porporatti, A. L., Flores-Mir, C., \& De Luca Canto, G. (2019). Prevalence of dentofacial injuries among combat sports practitioners: a systematic review and meta-analysis. Dental Traumatology, 36, 124-40.

Ribeiro, A. P. F., Tozzi, T. C. F., Tumelero, S., Carrijo, G. A. N., Vieria, L. R., Loureiro, C., \& Jacinto, R. C. (2021). Knowledge and prevalence of trauma and evaluation of the impact of educational and preventive actions on a population of athletes. Research, Society and Development, 10(4), doi:10.33448/rsdv10i4.13913.

Schildknecht, S., Krastl, G., Kuhl, S., \& Filippi, A. (2012). Dental injury and its prevention in Swiss rugby. Dental Traumatology, 28, 465-9.

Sepet, E., Aren, G., Dogan Onur, O., Erdem, A. P., Kuru, S., Tolgay, C. G., \& Unal, S. (2014). Knowledge of sports participants about dental emergency procedures and the use of mouthguards. Dental Traumatology, 30, 391-5.

Siewe, J., Rudat, J., Zarghooni, K., Sobottke, R., Eysel, P., Herren, C., Knoll, P., Illgner, U., \& Michael, J. (2015). Injuries in competitive boxing. A prospective study. Int J Sports Med, 36, 249-53.

Singh, G., Garg, S., Damle, S.G., Dhindsa, A., Kaur, A., \& Singla, S. (2014). A study of sports related occurrence of traumatic orodental injuries and associated risk factors in high school students in north India. Asian J Sports Med, 5, 1-5.

Tewari, N., Johnson, R. M., Mathur, V. P., Rahul, M., Goel, S., Ritwik, P., Bansal, K., \& Atif, M. (2021). Global status of knowledge for prevention and emergency management of traumatic dental injuries in sports persons and coaches: A systematic review. Dental Traumatology, 37(2), $196-207$.

Tiryaki, M., Saygi, G., Yildiz, S. O., Yildirim, Z., Erdemir, U., \& Yucel, T. (2017). Prevalence of dental injuries and awareness regarding mouthguards among basketball players and coaches. The Journal of sports medicine and physical fitness, 57(11), 1541-1547.

Tiwari, V., Saxena, V., Tiwari, U., Singh, A., Jain, M., \& Goud, S. (2014). Dental trauma and mouthguard awareness and use among contact and noncontact athletes in central India. Journal of oral science, 56(4), 239-243.

Tulunoglu, I., Ozbek, M. (2006). Oral trauma, mouthguard awareness, and use in two contact sports in Turkey. Dental Traumatology, 22, 242-6. 
Research, Society and Development, v. 10, n. 6, e57810616133, 2021

(CC BY 4.0) | ISSN 2525-3409 | DOI: http://dx.doi.org/10.33448/rsd-v10i6.16133

Tuna, E. F., \& Ozel, E. (2014). Factors affecting sports-related orofacial injuries and the importance of mouthguards. Sports medicine, 44(6), 777-783.

Vucic, S., Drost, R. W., Ongkosuwito, E. M., \& Wolvius, E. B. (2016). Dentofacial trauma and players' attitude towards mouthguard use in field hockey: a systematic review and meta-analysis. Br J Sports Med, 50(5), 298-304. 